\title{
Atenção primária à saúde: percepções de enfermeiros/as frente à violência sexual contra crianças e adolescentes
}

\author{
Primary health care: perception of the nurses in the face of the sexual violence against children and teenagers
}

Atención primaria de salud: percepciones de enfermeros frente a la violencia sexual contra niños, niñas y adolescentes

\begin{abstract}
RESUMO
Objetivo: Compreender a percepção dos enfermeiros(as) que atuam na Atenção Primária à Saúde (APS) acerca da violência sexual contra crianças e adolescentes. Método: Trata-se de um estudo descritivo, exploratório com abordagem qualitativa, realizado com doze enfermeiros(a) atuantes nas APS da zona urbana e rural do município de Iguatu, Ceará. Resultados: Dez dos participantes eram do gênero feminino (83\%), e dois do masculino (17\%), variando entre 30 a 41 anos, mantendo-se constante entre 30 a 35 anos de idade (42\%). Notou-se uma certa confusão acerca da violência ética e moral pelos profissionais atuantes, todavia, os mesmos referem que estão aptos a atender crianças e adolescentes em situações de violência, e que a acessibilidade aos serviços/redes é uma potencialidade da APS. Conclusão: Constatou-se que a percepção dos enfermeiros(a) ficou clara no decorrer do estudo, bem como seus conhecimentos e ações voltadas ao enfrentamento da violência sexual contra crianças e adolescentes.
\end{abstract}

DESCRITORES: Atenção Primária à Saúde; Estratégia Saúde da Família; Abuso Sexual; Criança; Enfermagem.

\section{ABSTRACT}

Objective: This research aims to understand the perception of nurses that act on the Primary Health Care (PHC) regarding the sexual violence against children and teenagers. Method: This is a descriptive and exploratory study with a qualitative approach, realized with 12 nurses acting of the urban and rural zones of Iguatu state of Ceará. Results: 10 of the participants are female, about $(83 \%)$ and only 2 are male $(17 \%)$, varying between 30 and 41 years old, maintaining itself constant between 30 and 35 years old $(42 \%)$. It was noticed that there is a certain confusion regarding the ethic and moral violence acting professionals. However, they refer that they are able to take care of children and teenagers in situations of violence and that the accessibility to the services is potentiality to the PHC. Conclusion: It was observed that the perception of the nurses was made clear during the study, as well as their knowledge and actions which were oriented to the confrontation of the violence against children and teenagers.

DESCRIPTORS: Primary health Care; Family health strategy; Sexua abuse; Children; Nursing.

\section{RESUMEN}

Objetivo: La investigación busca comprender la percepción de los enfermeros que actúan en la Atención Primaria de Salud (APS) acerca de la violencia sexual contra niños, niñas y adolescentes. Método: Se trata de un estudio descriptivo, exploratorio con abordaje cualitativa, realizado con 12 enfermeros (as) actuantes de la zona urbana y rural del municipio de lguatu, Ceará. Resultados: 10 de los participantes son del género femenino, alrededor de (83\%), y dos del masculino (17\%), que van de 30 a 41 años, manteniéndose constante entre 30 a 35 años de edad (42\%). Se notó que hay una cierta confusión acerca de la violencia ética y moral por parte de los profesionales actuantes, pero, refieren que están aptos para atender a niños, niñas y adolescentes en situaciones de violencia, y que el acceso a los servicios/redes es una potencialidad de la APS. Conclusión: Se constató que la percepción de los enfermeros quedó clara en el decorrer del estudio, bien como sus conocimientos y acciones orientadas al enfrentamiento de la violencia sexual contra niños, niñas y adolescentes.

DESCRIPTORES: Atención Primaria de Salud; Estrategia Salud de la Familia; Abuso Sexual; Niño y niña; Enfermería.

RECEBIDO EM: 15/09/2020 APROVADO EM: 21/10/2020 


\section{Kadson Araujo da Silva}

Discente do Curso Bacharel em Enfermagem pela Universidade Regional do Cariri-URCA. ORCID: 0000-0001-6397-0686

\section{Antonia Daila Martins de Souza}

Enfermeira pela Universidade Regional do Cariri-URCA. Bacharel em Enfermagem.

ORCID: 0000-0002-3961-1023

\section{John Carlos de Souza Leite}

Enfermeiro. Docente do Departamento de Enfermagem da Universidade Regional do Cariri-URCA. Mestre em Saúde da Criança e do Adolescente pela Universidade Estadual do Ceará-UECE.

ORCID: 0000-0002-0183-6913

\section{Riani Joyce Neves Nóbrega}

Enfermeira. Docente do Departamento de Enfermagem da Universidade Regional do Cariri-URCA. Mestre em Saúde da Criança e do Adolescente pela Universidade Estadual do Ceará-UECE.

ORCID: 0000-0002-6696-8298

\section{Marília Brito de Lima}

Enfermeira. Docente do Departamento de Enfermagem da Universidade Regional do Cariri-URCA. Mestre em Enfermagem pelo Programa de Pós-Graduação em Enfermagem- Universidade Federal do Ceará.

ORCID: 0000-0003-1797-2343

\section{João Paulo Xavier Silva}

Enfermeiro. Docente do Departamento de Enfermagem da universidade Regional do Cariri-URCA, Centro Universitário Vale do Salgado-UNIVS e do Centro Universitário DR. Leão Sampaio-Unileão. Mestre em Enfermagem pela Universidade Regional do Cariri-URCA.

ORCID: 0000-0003-3082-9373

\section{INTRODUÇÃO}

A violência é compreendida como o uso de força física e/ou ameaças aplicadas a si próprio ou contra outras pessoas, resultando em danos psicológicos, lesões, e em alguns casos a morte. Logo, caracteriza-se como um fenômeno social complexo e multívoco, acometendo diversos grupos sociais, dentre eles as crianças e os adolescentes ${ }^{(1)}$.

Segundo o Estatuto da Criança e do Adolescente (ECA) $(1990)^{(2)}$, é considerado criança o indivíduo de até 12 anos de idade incompleto e adolescente de 12 a 18 anos. Existem inúmeras maneiras de violentar uma criança, a saber: o abuso físico, psicológico, abuso sexual e a negligência, sendo as formas mais comuns de violência voltadas a este público ${ }^{(3)}$.

No Brasil, entre os anos de 2011 a 2017, observou-se um aumento de $83 \%$ nas notificações de violência. Nesse período, foram notificados 184.524 casos, sendo $31 \%$ voltados à criança e $45 \%$ ao adolescente. No
Ceará, entre janeiro e junho de 2017, foram registradas duas mil denúncias representando em média 11 por dia, confrontando com o ano de 2016, pode-se perceber que houve um aumento de $26 \%$ de ocorrências ${ }^{(4)}$.

Para o enfrentamento da violência à criança e ao adolescente, inúmeras entidades estão envolvidas nesse processo, podendo assim destacar o Centro de Referência de Assistência Social (CRAS), Centro de Referência Especializado de Assistência Social (CREAS), Ministério dos Direitos Humanos, Juizado de menores, Educação e Saúde, e a Atenção Primária à Saúde (APS) ${ }^{(5)}$.

Inserido na APS, o enfermeiro(a) atua como o mais próximo do contato familiar, possibilitando o reconhecimento precoce de situações que envolvam quaisquer métodos de agressões, logo, percebe-se que a atuação no enfrentamento a violência é ampla e complexa, em vista ao processo de enfermagem, identificação de sinais físicos e comportamentais, bem como ações educativas voltadas a família e comunidade, além da notificação compulsória ${ }^{(1)}$.

Portanto, o enfermeiro(a) faz-se necessário nas investigaçôes e nas ações de enfrentamento voltadas a este público, servindo de subsídio na elaboração de estratégias eficazes ao combate à violência.

Dito isso, a presente pesquisa teve como objetivo compreender a percepção dos enfermeiros(as) que atuam na APS acerca da violência sexual contra crianças e adolescentes.

\section{MÉTODO}

Trata-se de um estudo descritivo, exploratório com abordagem qualitativa, realizado com enfermeiros(as) que atuam na APS do município Iguatu-Ceará, sendo respectivamente sete da zona urbana e cinco da zona rural, totalizando 12 participantes.

Definiu-se como critérios de inclusão na pesquisa: os enfermeiros(as) com um período mínimo de seis meses de atuação na unidade, considerando a experiência no setor. E para os critérios de exclusão: ausência por licença, atestado ou durante coleta, 
bem como vulnerabilidade psicoemocional do participante.

Inicialmente realizou-se um teste piloto no próprio município onde ocorreu a coleta de dados, consequência da maior proximidade entre o local e o pesquisador. Ao final da aplicação do teste, executou-se alguns ajustes no questionário sociodemográfico para melhor caracterização das amostras dos participantes.

A coleta de dados foi realizada na própria Unidade Básica de saúde (UBS), marcada por ligação ou convite verbal definindo-se data e horário, nos meses de setembro e outubro de 2019, por meio de uma entrevista semiestruturada contendo indagações sobre a temática proposta, utilizando um gravador digital (smartphone) para a preservação das falas dos participantes.

Após a coleta, os dados foram organizados e digitalizados na íntegra, de modo a auxiliar na interpretação e análise dos dados e compará-los, posteriormente, com uma leitura minuciosa. Para análise estatística dos dados utilizou-se o software IRAMUTEQ (Interface de $\mathrm{R}$ pourles Analyses Multidimensionnelles de Textes et de Questionnaires), através de dois métodos de análises, a Classificação Hierárquica Descendente (CHD) para recuperar corpus original, e a Nuvem de Palavras (NP) para organização de vocabulários ${ }^{(6)}$, tendo as falas caracterizadas por letras e números, correspondendo cada participante.

Os mesmos foram informados quanto a relevância de sua contribuição para com o estudo, esclarecendo os objetivos e procedimentos da pesquisa, pelo qual confirmaram sua adesão através da assinatura do Termo de Consentimento Livre e Esclarecido (TCLE) visando garantir que os integrantes tivessem total liberdade de desistir a qualquer momento, e que suas identidades seriam preservadas utilizando-se de um

Tabela 1- Características sociodemográficas dos profissionais enfermeiros da Atenção Primária à Saúde do município de Iguatu. Iguatu, CE, 2019.

$\begin{array}{ccc}\begin{array}{c}\text { CARACTERISTICAS SOCIODEMOGRÁFICA } \\ \text { Identidade de gênero }\end{array} & \mathbf{N}^{\circ} & \% \\ \text { Mulher Cis } & 10 & 83 \% \\ \text { Homem Cis } & 2 & 17 \% \\ \text { Idade } & & \\ 30-35 & 5 & 42 \% \\ 36-40 & 3 & 25 \% \\ \text { Acima de 41 } & 4 & 33 \% \\ \text { Raça/cor } & & \\ \text { Branca } & 5 & 42 \% \\ \text { Parda } & 7 & 58 \% \\ \text { Renda familiar } & & 17 \% \\ \text { 2-3 salários mínimos } & 2 & 83 \% \\ \text { 3 ou mais } & 10 & \end{array}$

Tabela 2- Características profissionais dos enfermeiros da Atenção Primária à Saúde do município de Iguatu. Iguatu, CE, 2019.

$$
\begin{gathered}
\text { CARACTERÍSTICAS PROFISSIONAIS } \\
\text { Titulação máxima } \\
\text { Especialistas }
\end{gathered}
$$$$
N^{\circ} \%
$$

Recebeu capacitações na área de violência contra criança e adolescente? anonimato de seus nomes, aplicando-se pseudônimos como: E1, E2, E3, em que o E indica à palavra enfermeiro, seguido por numeração subsequente.

$\mathrm{O}$ estudo atendeu as determinações propostas pelo Conselho Nacional de Saúde através da Resolução $\mathrm{N}^{\circ} 466^{(7)}$, obtendo aprovação do Comitê de Ética em Pesquisa (CEP) da Universidade Regional do Cariri (URCA), sob parecer de número: 3.606.663 e com Certificado de Apresentação de Apreciação Ética (CAAE) correspondente a numeração: 21274319.9.0000.5055.

\section{RESULTADOS}

Participaram da pesquisa dez profissionais do gênero feminino correspondendo em cerca de (83\%) dos entrevistados e, apenas dois do gênero masculino (17\%). No que diz respeito à faixa etária dos participantes, observou-se uma variação entre 30 a 41 anos, mantendo-se constante entre 30 a 35 anos de idade (42\%).

Para descrever detalhadamente o perfil dos partícipes e as demais informações, foi preciso realizar um delineamento das características sociodemográficas e profissionais dos mesmos. Apresentando essas variáveis na tabela 1 e 2 , respectivamente:

A análise de dados foi constituída por um corpus expresso por um total de 12 textos gerados com base na transcrição das 12 entrevistas, originando assim 223 Segmentos de Textos (ST), proporcionando um aproveitamento de até 169 segmentos, o que equivale a $75,7 \%$. Surgiram assim 7.815 vocábulos distintos, 740 condizem com as palavras que foram citadas uma única vez no corpus (frequência igual a um) e no total foram 761 formas ativas com uma frequência $>=3$. De acordo com Camargo e Justus (2018) para que se tenha um progresso benéfico, o ideal é que o corpus seja compatível a um índice mínimo de 75\%.

Assim, os dados colhidos durante as entrevistas foram divididos em quatro classes, onde as palavras foram distinguidas por meio da Classificação Hierárquica Descendentes diante dos segmentos de texto que expóe em tamanhos diferentes, indicando tanto o grau 


\section{artigo}

Araujo da Silva, K.; Souza, A.D.M.; Leite, J.C.S; Nóbrega, R.J.N.; Lima, M.B.; Xavier Silva, J.P.;

Atenção primária à saúde: percepções de enfermeiros/as frente à violência sexual contra crianças e adolescentes

de associação, quanto a paridade no contexto das categorias elaboradas. Por sua vez, as classes foram originadas pelas palavras de maior importância, frequência e interligação com a classe x2 (qui-quadrado). Estas apresentadas no Dendograma da Figura 1 a seguir:

\section{Classe l: Compreensão dos enfermei- ros(as) acerca dos tipos de violência}

As palavras mais significativas foram: "sexual", "ato", "violência”, "físico", "direito", "abuso", "falta”, "psicológico”, "comum”, respeito", as quais compreendem a concepção dos enfermeiros sobre violência, destacando-se nas falas:

(...) É, violência é qualquer tipo de agravo que se imponha contra a vontade do outro. Qualquer tipo de ato, não praticamente o praticado, mas qualquer ato, ou tentativa, ou sugestão sexual. (E12).

(...) É tudo aquilo que é.... roubado, deturpado dos nossos direitos, no caso violentado dos nossos direitos, existe a violência sexual, a violência moral, física, de um modo geral existem vários tipos de violência como falta de ética, enfim (E2).

\section{Classe II: Desafios e potencialidades da APS}

Figura 1 - Dendograma da Classificação Hierárquica Descendente com as divisões e conteúdo corpus da pesquisa. Iguatu, CE, Brasil, 2019.

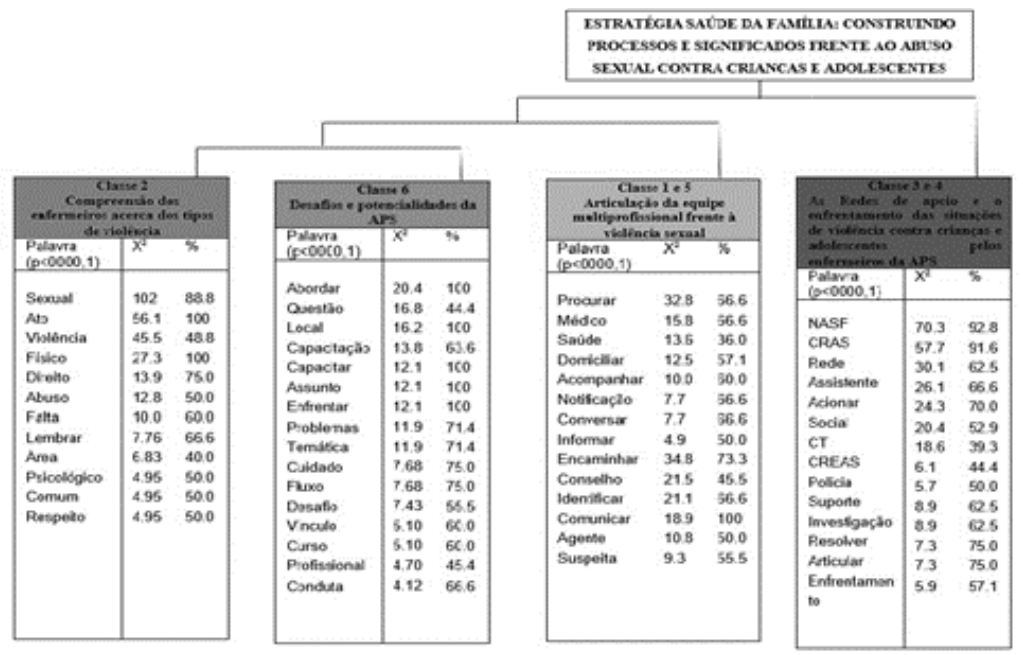

As palavras mais evidentes nessa classe foram: "abordar", "questão", "local”, “capacitação", "enfrentar", "problemas”, "temática”, "cuidado", "fluxo", "desafio", "vínculo” e "profissional", caracterizando os possíveis desafios e potencialidades, destacados nos discursos.

(...) Eu acho que um desafio a ser enfrentado é justamente essa questão da capacitação, porque como você já perguntou aí, eu não lembro de ter recebido nenhum tipo de capacitação voltada à essa temática, uma coisa que deve ser trabalhada. (E10).

(...) Desafio é exatamente aquilo que eu falei, que se encontra, não tem apoio policial, então tá dentro da área, envolver questões de violência, envolve questões sociais, envolve coisas que não tem como resolver, $e$ às vezes fica de mão atadas, tenta acionar as outras redes, mas nem sempre tem sucesso (E09).

\section{Classe III: Articulação da equipe mul- tiprofissional frente a violência sexu- al contra crianças e adolescentes}

"As palavras que mais se destacaram nessa classe foram: "suspeita", "médico", "saúde", "domiciliar", “ acompanhar", "notificação", "conversar", "informar", "encami- 
for o caso. Então, mexe com toda a rede, isso vai muito depender do fato, e das condiçôes daquela criança (E9).

\section{Nuvem de Palavras}

Ao fazer uma análise geral da NP apresentada na Figura 2 da temática da percepção dos enfermeiros acerca do abuso sexual contra crianças e adolescentes, a palavra "violência", "criança", "sexual", "conselho", são a palavras mais evidentes e de maior relevância no corpus textual.

As falas processadas no software que constituíram a NP estão explicitas nos recortes a seguir:

(...) Olha na violência contra a criança e o adolescente são os conselhos que a gente mais conbece é 0 conselho do adolescente, conselho da criança e do adolescente e do idoso, os outros assim a gente não sabe muito como manejar não. (E1).

(...) A violência sexual não é só aquela que você ver uma pessoa pegar e estuprar e tudo, as vezes a violência sexual é dentro de casa também ... é quando a esposa não permite ou qualquer um, uma mulher ou um homem não permite e é forçado. (E3).

Deste modo, o conhecimento dos profissionais enfermeiros(a) sobre os aspectos e conceitos a respeito da violência sexual, evidenciou-se uma gama de informações sobre a temática, e isso implica diretamente na qualidade do cuidado. Entretanto, é necessário ofertar melhores condições de trabalho e promoção de capacitações que auxiliem o atendimento fornecido.

\section{DISCUSSÃO}

Compreende-se que os enfermeiros(as) têm um conhecimento prévio sobre os tipos de violência e o conceito de violência sexual contra crianças e adolescentes. Entretanto, é notório nas falas que muitos confundem alguns termos e sua aplicabilidade, como violência ética e moral ${ }^{(8)}$.

As situações de violência à criança e ao adolescente são veladas por relações de poder, por este motivo, constituem o público mais suscetível à violência sexual, justificado pelo medo ou vínculos afetivos importantes com seus agressores, fazendo com que estes não consigam distinguir as possibilidades de lidar com o abuso, inclusive com o poder de dizer não( ${ }^{(9)}$.

Quando se trata da violência sexual a este público, faz-se necessário identificar todas as variáveis relacionadas a esse fenômeno, como aspectos culturais, sociais, econômicos e o ambiente que a vítima e família estão inseridas, pois contribuem para o início e propagação do ciclo geracional ${ }^{(10)}$.

Assim, segundo Ortega et al. ${ }^{(11)}$, o âm-

Figura 2 - Nuvem de palavras gerada pelo software IRAMUTEQ. Iguatu, CE, Brasil, 2019.

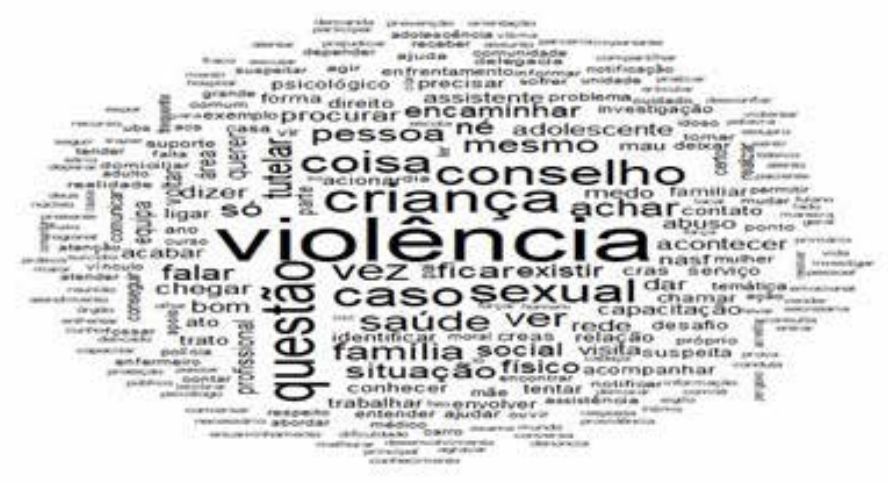

bito da saúde encontra-se em um contínuo processo de renovação e avanços tecnológicos e de conhecimento. Por isso, faz-se necessário que os profissionais da saúde, em particular os enfermeiros, estejam frequentemente em busca de atualizações e capacitações enquanto formação profissional, com o objetivo de fornecer uma assistência de qualidade e embasada em evidências científicas. Corroborando para com a erradicação das situações dos contextos de violência sexual ou de qualquer natureza, enfatizando o papel indispensável do enfermeiro na linha de frente a este combate.

Um estudo realizado por Galino et al. ${ }^{(12)}$, confirma que é fundamental que os enfermeiros(as) conheçam todos os tipos de violência provendo identificação precoce, assistência precisa voltada a cada caso, bem como realizar ações de prevenção e desestímulo à violência. Nesta perspectiva, os enfermei$\operatorname{ros}($ as) do presente estudo referem que estão aptos a atender crianças e adolescentes em situações de violência, e que a acessibilidade aos serviços/redes é uma potencialidade da APS. Assim, ratificamos a importância de se ter profissionais comprometidos e éticos, onde fatores limitantes da sua atuação não comprometerão a suas atribuições.

Destarte, a qualificação profissional pode possibilitar uma melhoria nas condutas voltadas ao cliente em situação de vulnerabilidade às questôes que envolvam violência, enaltecendo a atuação do enfermeiro na APS, levando em consideração as diversas habilidades desenvolvidas nesse período de ganho de conhecimento. Diante disso, percebe-se que a educação continuada não presente, pode atenuar na prevenção da violência seja ela repetitiva e/ou de novas ocorrências, sendo prioritariamente indispensável a detecção logo após o primeiro contato com a vítima ${ }^{(13)}$.

Nessa perspectiva, a identificação de situações de violência caracteriza-se como a fase mais importante na atuação da equipe na APS. Um estudo, realizado com uma equipe de enfermagem de uma UBS na região Centro Sul Fluminense refere que para identificar um caso de violência o profissional precisa ser sensível, ter habilidade e está disponível para ouvir atentamente 


\section{artigo}

Araujo da Silva, K.; Souza, A.D.M.; Leite, J.C.S; Nóbrega, R.J.N.; Lima, M.B.; Xavier Silva, J.P.

Atenção primária à saúde: percepções de enfermeiros/as frente à violência sexual contra crianças e adolescentes

o discurso do outro ${ }^{(10)}$. Sendo assim, as orientações e suporte aos familiares devem ser pautados no acolhimento, esclarecimento sobre a importância das atitudes dos pais, tanto na prevenção a violência como na tomada de decisões, objetivando o cuidado sistematizado e integral ${ }^{(14)}$.

Portanto, o enfermeiro(a) torna-se imprescindível na assistência em situações de violência, associado ao caráter cientifico no cuidado individual a vítima de violência e sua família. Deve-se levar em consideração a maneira de condução do atendimento, de modo que seja um espaço favorável para interação e o desenvolvimento de vínculo para com o usuário ${ }^{(15)}$.
Sendo assim, impreterível refletir sobre o estímulo de discussões sobre a temática em pauta e realizar capacitações para com os profissionais de saúde, focalizando o enfermeiro por desempenhar um papel de relevância na identificação e notificação dos casos de violência. Posto isso, compreende-se que a temática deve ser incluída no contexto acadêmico, para que os discentes e futuros profissionais se formem conscientes de seu papel, reconhecendo sua contribuição para a comunidade voltada a situações violência.

\section{CONCLUSÃO}

A percepção, dos conhecimentos e ações dos enfermeiros(as) ficou clara no decorrer do estudo quanto ao enfrentamento da violência sexual contra crianças e adolescentes. Levando em consideração que APS exerce um papel fundamental para enfrentar o ciclo da violência, afirmando a necessidade de ressignificar as práticas em saúde e consolidar políticas voltadas a temática em questão.

Diante disso, reflete-se sobre a relevância de capacitações para estes profissionais e discussões sobre a problemática. Contudo, ainda há uma lacuna na condução de casos de violência, com isso, o presente estudo propagará novos conhecimentos acerca da temática e ampliará novos horizontes.

\section{REFERÊNCIAS}

1. Teixeira SO. Atuação da enfermagem frente ao abuso sexual de crianças e adolescentes: pesquisa integrativa [Monografia] [Internet]. Brasília: Uniplac; 2019. 19 p. [acesso 2020 jul 7]. Disponivel em: https://dspace.uniceplac.edu.br/bitstream/123456789/317/1/Shirniara_Texeira_003424.pdf

2. Brasil. Lei no 8.069. de 13 de julho de 1990. República Federativa do Brasil. Brasília: Senado Federal; 1990.

3. Ministério dos direitos humanos (Brasil). Ações de Proteção a Crianças e Adolescentes contra violências: levantamentos nas áreas de saúde Assistência social. Turismo e direitos humanos. Brasília (DF): Oministério; 2018.

4. Ministério da saúde (Brasil). Análise epidemiológica da violência sexual contra crianças e adolescentes no Brasil. 2011 a 2017. Brasília (DF): 0 ministério; 2018.

5. Ministério da saúde (Brasil). Linha de cuidado para a atenção integral à saúde de crianças, adolescentes e suas famílias em situação de violências: orientação para gestores e profissionais de saúde. Brasília (DF): 0 ministério; 2014.

6. Camargo BV, Justo AM. IRAMUTEQ: um software gratuito para análise de dados textuais. Temas Psicologia [Internet]. [Ribeirão Preto]. 2013 set [acesso 2020 jul 10]; 21(2): 513-518. DOl: http://dx.doi.org/10.9788/TP2013.2-16.

7. Ministério da saúde (Brasil). Resolução № 466. de 12 de dezembro de 2012. Comitê de ética em pesquisa humana. Brasília. (DF): 0 ministério; 2013.

8. Hohendorf JV, Patias ND. Violência sexual contra crianças e adolescentes: identificação, consequências e indicações de manejo. Barbarói [Internet]. [Santa Cruz do Sul] 2017 set [acesso 2020 jul 10]; 49: 239-257. DOl: http://dx.doi.org/10.17058/ barbaroi.v0i49.9474.

9. Ministério da saúde (Brasil). Proteger e cuidar da saúde de adolescentes na atenção básica. Brasília (DF): O ministério; 2017.

10. Lobato GR, Maraes CL, Nascimento MC. Desa os da atenção à violência doméstica contra crianças e adolescentes no Programa Saúde da Família em cidade de médio porte do Estado do Rio de Janeiro, Brasil. Rev. Caderno de Saúde Pública [Internet]. [Rio de Janeiro]. 2012 set [ acesso 2020 jul 13] V.28. n. 9. P.17491758. Disponivel em: https://www.scielosp.org/article/ssm/ content/raw/?resource_ssm_path=/media/assets/csp/v28n9/ v28n9a13.pdf.

11. Ortega MDCB, Cecagno D, Llor MAS, et al. Formação acadêmica do profissional de enfermagem e sua adequação às atividades de trabalho. Revista Latino-Americana em Enfermagem [Internet]. [Ribeirão Preto]. 2015 set [acesso 2020 out 16]; 23(3): 404-410. Disponível em: https://www.scielo.br/ scielo.php?script=sci_arttext\&nrm=iso\&lng=pt\&tlng=pt\&pid =S0104-11692015000300404.

12. Galino NAL, Gonçalves CFG, Neto NMG, et al. Violência infanto-juvenil sob a ótica da enfermagem. Rev enferm UFPE on line [Internet]. [Recife] 2017 set [acesso 2020 jul 16]; 11(Supl. 3): 1420-1429. DOI: 10.5205/reuol.10263-91568-1-RV. 1103 sup201714.

13. Saraiva RJ, Rosas AMTF, Valente GSC, et al. Qualificação do enfermeiro no cuidado a vítimas de violência doméstica infantil. Ciencia y Enfermeria [Internet]. [Chile]. 2012 set [acesso 2020 out 16]; 18(1): 17-27. Disponivel em: https://www.redalyc.org/ pdf/3704/370441809003.pdf.

14. Soares EMR, Silva NL, Matos MAS, et al. Perfil da violência sexual contra crianças e adolescentes. Rev. Interd [Internet]. [local desconhecido]. 2016 set [acesso 2020 jul 13]; 9(1): 87-96. Disponivel em: https://revistainterdisciplinar.uninovafapi.edu.br/ index.php/revinter/article/view/754.

15. Moreira AC, Silva TASM. Cuidados de enfermagem as vítimas de violências interpessoais na atenção básica. Revista Pró-Universitário SUS [Internet]. [local desconhecido]. 2019 set [acesso 2020 jul 21]; 10(1): 42-46. DOI: https://doi.org/10.21727/rpu. v10i1.1650. 